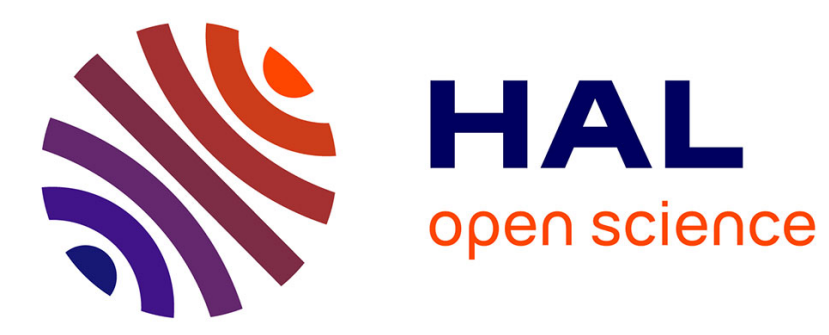

\title{
Diffusion des TIC et mobilité. Permanence et renouvellement des problématiques de recherche
}

\author{
Alain Rallet, Anne Aguilera, Caroline Guillot
}

\section{To cite this version:}

Alain Rallet, Anne Aguilera, Caroline Guillot. Diffusion des TIC et mobilité. Permanence et renouvellement des problématiques de recherche. Flux - Cahiers scientifiques internationaux Réseaux et territoires, 2009, 2009/2 (78), pp.7-16. halshs-00489835

\section{HAL Id: halshs-00489835 \\ https://shs.hal.science/halshs-00489835}

Submitted on 7 Jun 2010

HAL is a multi-disciplinary open access archive for the deposit and dissemination of scientific research documents, whether they are published or not. The documents may come from teaching and research institutions in France or abroad, or from public or private research centers.
L'archive ouverte pluridisciplinaire HAL, est destinée au dépôt et à la diffusion de documents scientifiques de niveau recherche, publiés ou non, émanant des établissements d'enseignement et de recherche français ou étrangers, des laboratoires publics ou privés. 


\title{
Diffusion des TIC et mobilité. \\ Permanence et renouvellement des problématiques de recherche
}

\author{
Alain Rallet*, Anne Aguiléra** et Caroline Guillot** \\ * Université Paris Sud, ANEIS \\ ** Université Paris Est, LVMT
}

\section{Résumé}

La question des liens entre la diffusion des outils de communication et la mobilité physique des individus n'est pas nouvelle et s'est posée avec l'arrivée du téléphone fixe, et, plus récemment, avec le développement d'Internet et du commerce en ligne. La spectaculaire diffusion d'outils de communication individuels et surtout portables, comme le téléphone mobile, a récemment donné un nouvel élan à cette thématique dans les champs de l'économie des transports, de la géographie et de la sociologie. Cet article propose une analyse critique des questionnements explorés par la littérature, en vue de mettre en avant les problématiques qui nous semblent devoir être mieux explorées.

\section{Summary}

The question of the relationship between the diffusion of communication tools and the physical mobility of individuals is not new and arose with the arrival of the fixed telephone and, more recently, the development of the Internet and especially the e-commerce. The extraordinary diffusion of individual and especially portable communication tools, like the mobile phone, has recently given a new impetus to this topic in the fields of transportation economics, geography and sociology. This article proposes a critical analysis of the questions that have been explored by the literature, in order to highlight the issues that we think should be better explored. 


\section{Introduction}

La question des liens entre le développement des outils de communication et la mobilité physique des individus n'est pas nouvelle (Massot, 1995 ; Mokhtarian, 2000). Elle s'est notamment posée avec l'arrivée du téléphone fixe dans les foyers (Claisse et Rowe, 1993), puis, plus récemment, avec le développement d'Internet et en particulier du commerce en ligne (Golob et Regan, 2001). La spectaculaire diffusion d'outils de communication individuels et surtout portables, comme le téléphone mobile, a récemment donné un nouvel élan à cette thématique dans les champs de l'économie des transports, de la géographie et de la sociologie. Cet article propose une analyse critique des questionnements explorés par la littérature récente, en vue de mettre en avant les problématiques qui nous semblent devoir être mieux explorées.

Notre principal constat est qu'en dépit des évolutions des propriétés et des usages des TIC, le questionnement dominant a très peu évolué. Il relève encore aujourd'hui majoritairement, en particulier dans le champ de l'économie des transports, d'une opposition entre effets de complémentarité voire d'induction, et effets de substitution. L'hypothèse de la complémentarité/induction soutient que l'usage croissant des TIC accompagne voire stimule la demande de déplacements. A l'inverse, les effets de substitution signifient que l'usage des TIC permet de réduire la demande de mobilité. Malgré de nombreux travaux empiriques, la réponse reste relativement indéterminée, même si la thèse de la complémentarité/induction domine (Rallet, 2000). Il serait difficile de soutenir le contraire car les enquêtes ne montrent pas de baisse sensible de la mobilité (sauf période particulière de fort renchérissement du coût de l'énergie). L'approche opposant complémentarité et substitution souffre toutefois de sérieuses limites. Elle s'intéresse généralement par nécessité (sinon la complexité est trop importante) à une seule technologie (par exemple le commerce en ligne), envisage des effets individuels (alors que probablement les effets au niveau des ménages sont tout aussi importants) sur un seul motif de déplacement (par exemple les achats), et ne considère en outre qu'une seule dimension de la mobilité (par exemple le nombre quotidien mais pas la distance, ou inversement) (Krizek et Johnson, 2003). La première partie de ce papier revient plus en détail sur ces travaux et explique leurs difficultés à rendre compte de la richesse et de la nature complexe des possibles interactions entre les TIC et la mobilité.

La seconde partie s'intéresse à une littérature plus récente mais plus prometteuse qui permet, nous semble-t-il, de dépasser avantageusement le débat entre complémentarité et substitution. L'approche adoptée, qui émane notamment de la sociologie et de la géographie du temps (Hägerstrand, 1970) et commence à pénétrer la socio-économie des transports (Schwanen et Kwan, 2008), envisage des interactions qualitatives plus riches, situées dans le domaine des pratiques spatio-temporelles et relationnelles des individus. Pour ces auteurs, ce n'est pas tant la relation entre l'usage des technologies et le nombre de déplacements qui est intéressante que le changement potentiel de nature de ces déplacements et de leur contexte via l'influence progressive de ces technologies sur nos modes de vie. Il s'agit entre autres de l'influence sur nos réseaux sociaux (privés et professionnels), sur le déroulement de nos activités dans le temps et dans l'espace, ainsi que sur notre façon de percevoir et de vivre les moments de notre mobilité. Ces auteurs défendent la thèse d'un enrichissement progressif des pratiques spatiales et temporelles par les TIC mobiles, autant qu'un usage des TIC mobiles à des fins de mobilité (ou plus largement de nomadisme) (Ling et Haddon, 2003). On ne cherche plus à savoir si les déplacements se transforment dans un sens ou dans un autre, mais on inverse la question : les déplacements s'organisent-ils différemment (selon les stratégies des individus) ? Il ne faut toutefois pas négliger les contreparties associées à ces évolutions, 
qui relèvent du contrôle des autres et par les autres des activités et notamment des déplacements de chacun d'entre nous.

\section{1- Substitution, complémentarité ou induction, une histoire sans fin}

La relation entre TIC et mobilité physique a longtemps été pensée de façon directe, c'est-à-dire relativement isolée d'autres paramètres et notamment des déterminants majeurs des comportements de mobilité (revenu, localisation résidentielle, activité, etc.). On a cherché à évaluer si l'usage des TIC allait amener les individus à réduire leur mobilité (1-1) ou au contraire à la soutenir voire à l'augmenter (1-2). Des attentes, parfois contraires, sont liées à cette évaluation. Ainsi, les politiques environnementales attendent des TIC une réduction des coûts de congestion et de pollution liés à la mobilité. Les TIC sont alors enrôlées dans la thématique du développement durable. Parallèlement, ces mêmes technologies sont souvent accusées d'affaiblir le lien social en incitant à la diminution des rencontres de face à face. Le débat complémentarité/substitution concentre ainsi d'importants enjeux sociétaux.

\section{1-1 La thèse de la substitution}

Elle s'appuie sur un imaginaire puissant, l'imaginaire spatio-temporel qui entoure le développement des TIC. Selon cet imaginaire, les télécommunications permettent de faire à distance ce que les individus faisaient à proximité et donc de substituer des relations virtuelles à des relations physiques. Alimentant des visions fantasmées du rôle des télécommunications dans les interactions spatio-temporelles, cet imaginaire a fait l'objet de nombreuses critiques (récemment Musso, 2008 sur le cyberespace). Il est toutefois sans cesse renouvelé par l'enrichissement spectaculaire et continuel des technologies de communication, de sorte qu'il fonctionne toujours comme arrière-plan de la thèse de la substitution. Selon cette thèse, l'usage des TIC amène les individus à diminuer le nombre de leurs déplacements.

D'une part les TIC peuvent inciter à effectuer, depuis des lieux fixes (notamment le domicile), une partie des activités qui nécessitaient auparavant des déplacements. Le télétravail, exercé à domicile ou dans des télécentres proches du domicile, est censé éviter les déplacements pour se rendre au travail. Depuis les années 70 , le thème du télétravail réapparaît de manière périodique en dépit d'attentes déçues. Le développement du commerce électronique lui a succédé au début des années 2000 avec la même approche (effectuer à distance des achats réalisés auparavant en magasin) et la même attente, résoudre les problèmes de mobilité urbaine (Rallet, 2005). Cette approche et cette attente se sont ensuite étendues à d'autres activités de service: la santé (télé-santé), l'administration (eadministration), l'enseignement (e-learning) mais aussi les loisirs (Mokhtarian et al., 2006).

D'autre part, ces technologies sont susceptibles de réduire les besoins de rencontres en face à face tant dans le domaine privé que professionnel (Lethiais et Aguiléra, 2007) en améliorant la qualité des relations à distance. Il existe en effet une panoplie variée de technologies de communication dont la combinaison flexible permet des interactions à distance de plus en plus riches. Les développements technologiques favorisent cette évolution avec la variété croissante des formes de communication (multimédia), leur nature de plus en plus informelle (emails, messageries instantanées, forums, réseaux sociaux, etc.) et la diminution de leur coût (ainsi le passage de la visioconférence de la salle dédiée au poste de travail).

Il n'est pas contestable et il est même trivial de soutenir que la communication à distance permet d'éviter certains déplacements. La question n'est pas là. Elle est celle des 
relations entre les télécommunications et la mobilité dans sa globalité. C'est ce que montre la littérature sur la complémentarité et l'induction.

\section{1-2 La thèse de la complémentarité et de l'induction}

Les TIC réduisent certains déplacements tandis que d'autres demeurent, certains le sont par nécessité (aller travailler) ou par choix (aller faire du shopping), alors que d'autres sont stimulés. Dans le domaine des achats, Internet peut être complémentaire des magasins (Rallet, 2001). Il sert beaucoup à se renseigner sur certains produits et leur disponibilité avant de se rendre au magasin. Il peut aussi faire découvrir des produits que l'on va ensuite aller acheter (Douma et al., 2004). La récente étude de S. Farag et al. (2007) a d'ailleurs montré qu'au Danemark les acheteurs en ligne faisaient en moyenne (toutes choses égales par ailleurs) plus de déplacements d'achats que ceux qui n'achetaient pas en ligne.

Dans le domaine professionnel, la substitution n'est pas non plus évidente : F. Douma et al. (2004) montrent que les TIC sont souvent utilisées avant de partir au travail et après le retour au domicile, pour répondre aux emails et préparer sa journée de travail ou la suivante. Cela permet éventuellement de décaler ses horaires de départ et de retour au domicile afin de diminuer ses temps de transport, mais pas de se déplacer moins. Le même constat vaut pour les déplacements professionnels (Arnfalk et Kogg, 2003). On peut l'étendre aussi au domaine privé, l'effet de substitution étant probablement au moins contrebalancé par l'élargissement des réseaux (en termes de nombre de contacts et de taille géographique) que permettent les TIC. J. Larsen et al. (2006) prévoient ainsi une multiplication des contacts et donc des déplacements à longue distance, tant que les coûts de la mobilité à longue distance restent bas, notamment grâce aux compagnies low cost.

Plus généralement, les TIC s'avérant incapables de reproduire avec la même qualité toutes les facettes du face à face, les interactions interpersonnelles continuent d'impliquer des relations de face à face (Rallet et Torre, 2008). A l'instar de F. Jaureguiberry (1994), F. Ascher (1995) et M. Guillaume (2000), on peut même soutenir que les TIC, par effet de compensation, confèrent une nouvelle valeur à la co-présence physique. Dès lors les mobilités physiques ne relèvent plus principalement de nécessités instrumentales mais pallient un déficit relationnel. Les échanges de face à face reviennent à signifier à l'interlocuteur qu'on lui accorde une attention particulière: ils répondent au désir de créer des relations d'intersubjectivité partagée. On ne peut dès lors s'attendre à une baisse des rencontres directes mais au contraire à leur augmentation : accroissant le nombre d'interlocuteurs et la taille des réseaux sociaux, les TIC engendrent des besoins de rencontres de face à face et donc des déplacements.

Par ailleurs, prétendre établir une relation «directe» entre l'usage des TIC et l'évolution du nombre des déplacements rencontre au moins quatre limites importantes :

il est difficile par ce biais de prendre en compte de possibles effets croisés (induction pour certains déplacements, substitution pour d'autres): on peut en effet potentiellement se déplacer moins pour tel motif mais plus pour un autre. C'est bien la conclusion de l'analyse théorique de P.L. Mokhtarian et al. (2006) sur les possibles effets des TIC sur les déplacements de loisirs. Les auteurs montrent que tout dépend des caractéristiques des individus et de celles des activités de loisirs, qu'ils déclinent selon pas moins de treize dimensions... Cette multiplicité des cas de figure rend compte de la quasi-impossibilité de trancher d'un point de vue purement quantitatif ;

les effets de long terme peuvent diverger de ceux mesurés à court terme, notamment parce que les individus se familiarisent avec ces technologies et peuvent être 
amenés à les utiliser de façon couplée, que le taux d'équipement progresse et que les fonctionnalités évoluent rapidement comme on le voit pour le téléphone mobile ;

la bonne échelle de mesure n'est pas forcément l'individu mais peut-être le ménage (effet possible sur la répartition des tâches et donc des déplacements entre les conjoints) ;

les évolutions observées de la mobilité peuvent dépendre de biens d'autres paramètres que les seuls TIC. On pense au coût du transport, aux stratégies de localisation des acteurs, etc. Or sur ces aspects les TIC ont un rôle au mieux modéré, et il est complexe et sans doute présomptueux d'isoler un impact spécifique des TIC sur le nombre de déplacements au quotidien, qui est au premier chef déterminé par des contraintes spatio-temporelles fortes (lieu et horaires de travail, localisation des commerces, etc.) (Green, 2002). Il est très difficile en tout cas de considérer qu'on raisonne bien « toutes choses égales par ailleurs ».

Compte-tenu de ces limites, il n'est pas surprenant que les travaux empiriques cherchant à mesurer si les TIC réduisent ou non la mobilité arrivent à des conclusions contradictoires (Massot, 2005), ou probantes seulement pour une frange très spécifique de la population, comme celle des cadres hypermobiles (Lenz et Nobis, 2007). L'intérêt de ces travaux réside plutôt dans la mise en évidence de la grande complexité des liens TIC-mobilité et, par rebond, dans la réflexion sur les marges de manœuvre dont disposent (ou pas) les individus et donc la société pour réduire la mobilité grâce aux TIC (est-ce une bonne chose de généraliser le télétravail ?, etc.).

En conclusion, la question des relations entre les usages des TIC et la mobilité doit être posée autrement que sous le seul angle de leur impact sur le nombre de déplacements. La question n'est pas illégitime mais on ne peut espérer la traiter comme une relation directe. Il faut au préalable la réinsérer dans une problématique plus large d'analyse des relations entre les TIC et les programmes d'activité des individus. Dans une première phase, les TIC ne bouleversent pas les pratiques préexistantes. Elles les équipent et, à ce titre, les facilitent, les accroissent, les élargissent, mais sans les transformer. L'émergence de nouvelles pratiques au sein du programme d'activités des individus n'intervient que dans des phases ultérieures (Belton et De Coninck, 2006). Objet d'usages innovants, les TIC associées à de nouvelles pratiques sociales modifient l'organisation quotidienne de leurs activités dans le temps et dans l'espace, leurs réseaux sociaux et professionnels, et enfin leur façon de se déplacer (notamment dans un contexte de portabilité croissante des TIC). Nous ne disposons pas encore du recul nécessaire pour caractériser ces évolutions. Mais nous devons construire un cadre d'analyse qui permette de saisir les évolutions émergentes et d'orienter ainsi notre attention vers des objets de recherche pertinents.

\section{2- Mobilité et continuum spatio-temporel}

Le problème de l'approche en termes de substitution est qu'elle pense le changement sous une forme radicale. Ainsi un monde de relations virtuelles se substituerait à un monde de relations physiques. Exploitant les potentialités des technologies, les changements radicaux postulés sont mis en œuvre dans le cadre d'expérimentations, c'est-à-dire dans des conditions artificielles non pertinentes pour leur transposition dans la réalité sociale. Aussitôt affirmée, la thèse de la substitution secrète la thèse contraire : rien ne change radicalement (les relations de face à face sont toujours nécessaires), le seul effet étant d'amplifier la réalité existante (le nombre de déplacements). La problématique substitution/complémentarité est ainsi liée à une certaine vision du changement initié par les TIC. Pour en sortir, il faut modifier cette vision. Le changement s'effectue au départ lentement car il touche à des innovations sociales ou 
organisationnelles toujours lentes à se mettre en place. Le changement est d'abord graduel, la réalité existante étant simplement "augmentée » de l'usage des technologies sans rupture qualitative dans les activités et les modes de vie. Cependant la familiarisation avec les technologies, leur usage croissant et les phénomènes d'apprentissage qui leur sont liés finissent par les banaliser. C'est alors que peuvent apparaître des innovations sociales majeures. Les changements qualitatifs naissent paradoxalement de la banalisation des technologies alors qu'on nous présente souvent le schéma inverse (les innovations induites par des phénomènes de rupture technologique).

On est conduit ainsi à se demander quel est le type de banalisation technologique en cours susceptible de modifier de manière importante notre rapport à l'espace et nos pratiques de mobilité. Il s'agit de l'utilisation d'outils portables de traitement de l'information et de communication dans le contexte d'un espace géographique équipé de façon maillée d'interfaces de rencontres physiques et de connexions virtuelles (2-1). Même s'il ne se matérialise pas immédiatement par des innovations majeures, l'usage de ces outils se diffuse rapidement et ouvre la voie à des transformations de notre rapport à l'espace. Dans une seconde section, nous tentons d'esquisser le sens probable de ces transformations. Nous en déduisons quelques pistes de recherche sur les questions de la mobilité (2-2).

\section{2-1 Equipement maillé de l'espace en interfaces de rencontres physiques et virtuelles et apprentissage de nouvelles normes de communication}

Les années 80 ont été dominées par l'avènement des micro-ordinateurs. Ceux-ci ont radicalement modifié le caractère de l'informatique, autrefois centralisée, aujourd'hui décentralisée. Le micro-ordinateur s'est banalisé au sens où nous avons appris à exploiter et à faire usage de terminaux intelligents. Les années 90 ont été dominées par l'avènement d'Internet, c'est-à-dire la mise en réseau généralisée de l'informatique décentralisée. Il a été d'abord exploité comme simple outil de communication ou d'information avant de révéler ses propriétés intrinsèques de réseau social (peer-to-peer, réseaux sociaux numériques...) qui donnent aujourd'hui lieu à de véritables innovations sociales. Les années 2000 sont caractérisées par le développement des outils mobiles de communication. Ces outils ne se limitent pas au téléphone mais touchent un nombre croissant d'objets (ordinateurs, unités de stockage, lecteurs audio et vidéo, consoles de jeux....). Au caractère décentralisé de l'informatique et à la mise en réseau s'ajoute la portabilité de l'informatique. Cette portabilité est destinée à s'étendre à l'ensemble des objets avec l' «Internet des objets », chaque objet étant rendu « intelligent» et connectable avec d'autres objets par l'introduction d'une puce. D'ores et déjà, le développement du téléphone mobile qui intègre de plus en plus de fonctions, de l'ordinateur portable qui se fait de plus en plus petit et des terminaux dédiés (baladeurs musicaux, consoles de jeux, appareils photos numériques...) nous familiarise avec cette portabilité et s'intègre de manière croissante dans les pratiques sociales (travail, loisirs, transports...).

Les usages actuels de cette portabilité n'ont pas encore modifié de manière substantielle nos pratiques sociales et notre rapport à l'espace et à la mobilité. Ainsi les usages que font les individus du téléphone portable se traduisent plutôt par un enrichissement des pratiques existantes de coordination. Les travaux du COST 298 (Aguiléra, Guillot et Bonin, 2009) montrent que i) les adolescents s'adonnent à l'hypercoordination (le téléphone portable leur sert à intensifier encore plus leurs relations avec leur réseau de pairs et essaient d'être plus autonomes, ii) les parents (notamment les mères d'enfants en bas âge) font de la microcoordination et s'assurent que les enfants sont bien rentrés ou gardent des liens avec les enfants en cas de divorce et iii) les personnes âgées insistent davantage sur la sécurité que leur 
procure le téléphone portable. Dans le domaine professionnel, les outils mobiles facilitent le pilotage des employés déjà nomades mais ne se sont pas encore traduits par une nouvelle approche de la mobilité au sein des entreprises (Moeller, 2006). Donc, rien de véritablement révolutionnaire, l'usage des outils mobiles accentue des pratiques déjà existantes. Mais de nouveaux dispositifs d'équipement de l'espace se mettent en place au travers de ces pratiques pour l'instant peu spectaculaires. Une fois mis en place et leurs usages développés, ces dispositifs serviront de support à de nouvelles pratiques, à des innovations dans notre rapport à l'espace et à la mobilité.

Quels sont ces dispositifs d'équipement de l'espace ? Ils consistent à recomposer l'espace en un complexe hybride de relations virtuelles et de relations physiques. Les interactions sociales s'appuient sur des infrastructures dispersées dans l'espace géographique qui permettent d'articuler des relations physiques (de face à face) et des relations virtuelles (co-présence sans contact physique). La problématique de la substitution s'appuie sur la thèse qu'il y aurait un espace physique et un espace virtuel dont il s'agirait d'analyser les relations. Or il n'y a qu'un seul espace: un espace géographique équipé d'interfaces physiques (lieux d'interactions physiques) et d'interfaces virtuelles (lieux d'interactions virtuelles). Tout espace est une intrication de lieux de relations physiques ou/et virtuelles (les deux pouvant être confondus comme lorsqu'on envoie des emails à son voisin de bureau ou que l'on se sert de lieux comme les cybercafés pour rencontrer des gens).

Cette hybridation n'est pas en soi un phénomène nouveau puisqu'elle commence avec le téléphone et s'est amplifiée avec l'Internet fixe. Mais elle était limitée par i) la nature fixe des lieux de connexion virtuelle et ii) la nature plutôt pauvre de la communication à distance jusqu'à l'étape récente d'Internet (multimédia). La portabilité de l'informatique communicante étend considérablement l'équipement hybride de l'espace en lieux de relations physiques et virtuelles. Faisons l'analogie avec les moyens de se déplacer. La révolution de l'automobile a été de démultiplier les zones pouvant bénéficier de déplacements rapides grâce d'une part, à l'individualisation du moyen de transport et d'autre part, à sa plus grande capacité de se déployer dans l'espace alors que le chemin de fer contraignait beaucoup plus fortement les déplacements par son caractère collectif et sa plus grande rigidité. Toutes choses égales par ailleurs, l'Internet mobile est à l'Internet et au téléphone fixe ce que fut l'automobile au chemin de fer et à la diligence. Or la diffusion généralisée de l'automobile a fini par engendrer de nouveaux schémas spatiaux (Dupuy, 1995).

Avec les outils mobiles, les individus expérimentent aujourd'hui à large échelle de nouvelles habitudes de communication dans l'espace, même si elles ne se traduisent pas encore par de réelles innovations sociales. Par exemple, téléphoner dans la rue en marchant semblait incongru il y a quelques années. Ce n'est pas une attitude « naturelle », sauf pour les individus technophiles toujours à l'affût de nouvelles pratiques comportementales. Mais l'on observe que les gens y compris ceux résistants à cette pratique finissent par l'adopter car elle est une conséquence quasi mécanique de ce que le téléphone portable est désormais individualisé et un compagnon de leurs déplacements quotidiens parce que situé dans leur poche. Les gens vont donc répondre à des appels qu'ils reçoivent dans la rue ou en émettre pour exploiter un temps «libre». L'usage finit par se généraliser. La rue n'est plus alors un espace soustrait à la pratique de communication auparavant réservée à des lieux singuliers (domicile, travail, cabine publique pour des usages précis tel que l'émission d'appels contraints). Dans un premier temps, cette nouvelle pratique de communication (nouvelle parce que nouvellement inscrite dans l'espace) ne se traduit pas nécessairement par des innovations majeures dans les pratiques sociales et les activités des individus. Elle sert surtout à favoriser la micro-coordination des individus dans le cadre de leurs pratiques habituelles. Ainsi, au début de la diffusion du téléphone portable, certains utilisateurs répondaient aux enquêtes sur 
les avantages apportés par cet outil en déclarant qu'il leur permettait de ne plus mémoriser le code des immeubles puisqu'ils avaient maintenant le moyen d'interroger leurs amis au bas de l'immeuble. L'usage n'est pas bouleversant mais l'individu apprend à utiliser le téléphone dans une situation qui n'est pas «naturelle » pour lui. Il se répand ainsi une nouvelle norme sociale de communication (téléphoner dans la rue) qui ouvre la voie à des innovations susceptibles de transformer à terme notre relation à l'espace. Le téléphone portable devient notamment pour l'utilisateur un moyen de navigation physique et pour ses interlocuteurs un moyen de le suivre dans l'espace. Il permet ainsi de s'orienter différemment dans ses déplacements et de bénéficier de services de géolocalisation. Il y a bel et bien innovation, dont on peut attendre à terme de nouvelles façons de s'inscrire et de se déplacer dans l'espace. Il est prématuré d'anticiper l'impact sur la mobilité mais le fait qu'elle soit désormais équipée d'outils portables de communication est de nature à la transformer.

\section{2-2 L'espace comme continuum}

L'équipement maillé de l'espace en lieux d'interactions physiques et de connexions virtuelles que nous appelons l'hybridation de l'espace a deux impacts complémentaires sur l'organisation spatiale. D'une part, il permet la disjonction de lieux qui étaient auparavant situés dans un même endroit. Déjà le téléphone avait favorisé l'éclatement dans l'espace des fonctions de l'entreprise (Chandler, 1956). Cette disjonction s'étend aujourd'hui aux activités de services (enseignement, santé, administration...) alors qu'une des caractéristiques des services était l'unité de lieu de leur production et de leur consommation. La disjonction s'étend aussi à certaines interactions sociales (développement de formes de sociabilité à distance avec les réseaux sociaux numériques). D'autre part, les TIC relient les lieux disjoints et, de manière complémentaire aux déplacements, assurent la coordination rendue nécessaire par la disjonction des lieux. Mais jusqu'au développement des réseaux mobiles de communication, cette disjonction continuait de reposer sur le caractère discontinu de l'espace. Les TIC assuraient des formes de co-présence entre des lieux spécifiés, contraints par le caractère fixe des connexions (domiciles, lieux de travail, magasins....).

Les réseaux mobiles de communication assurent une plus grande continuité spatiotemporelle. Les individus deviennent potentiellement joignables en tout lieu et tout moment, sous la forme de connexions en situation nomade (possibilité de se connecter en déplacement mais sans être physiquement mobile à l'instant de la communication) ou de mobilité (en se déplaçant physiquement). Même si des zones demeurent non ou mal couvertes, comme on en fait parfois l'expérience au cours d'un déplacement, l'espace tend à devenir un continuum spatio-temporel au sens où les oppositions binaires qui caractérisaient l'espace physique (ici/ailleurs, proche/distant, présent/absent) sont relativisées. Car on ne cesse d'être en contact avec ses interlocuteurs même si l'on s'éloigne d'eux et ceci sans attendre d'arriver au lieu de destination. La co-présence n'est plus l'apanage de la seule proximité physique ou d'une relation virtuelle entre des lieux fixes. L'espace en ses divers lieux offre des formes graduées de co-présence selon la combinaison utilisée de lieux physiques et de connexions virtuelles pour établir l'interaction. Naturellement la co-présence réalisée dans une relation de face à face est à priori plus riche en interactions que le suivi d'une conversation par SMS durant un déplacement. Mais même par le biais d'une forme dégradée de co-présence comme l'envoi de SMS, l'interaction est maintenue. Ainsi l'équipement de plus en plus maillé de l'espace en combinaisons différenciées de moyens de transport et de communication mobiles offre de nouvelles perspectives à la réalisation d'activités spatialement disjointes comme communiquer, s'informer, se coordonner, effectuer une transaction, renforcer un lien social....

Plusieurs questions se posent alors qui sont autant de points de recherche : 
1) Quelles décompositions spatio-temporelles des activités vont résulter de la mise en continuité relative de l'espace?

Les individus doivent articuler deux choses :

- les dotations inégales de l'espace en possibilités de se déplacer et de communiquer car même si l'espace est marqué par une certaine continuité, la possibilité d'établir ou de maintenir une interaction n'est pas la même en tout lieu et en tout moment. Il faut ajuster le déroulement de l'interaction selon les potentialités du lieu et du moment ;

- la nature des activités et des interactions qui les sous-tendent. Les activités sont caractérisées par des intensités interactionnelles plus ou moins fortes. Ce n'est pas la même chose de négocier une soirée avec des amis et de négocier une transaction commerciale, de piloter des trajets et de dérouler un processus de décision.

Les individus devront apprendre à apparier un espace différencié en possibilités de connexion et les caractéristiques des activités de façon à définir une décomposition spatiotemporelle adéquate de ces activités. Un effet envisageable en matière de mobilité physique est l'accroissement des pratiques de chaînage, c'est-à-dire d'enchaînement des déplacements sans (re)passer par le domicile (Srinivasan et Raghavender, 2005). On peut plus largement envisager la mise en place, subie ou sous contrainte, de formes d' «optimisation » de la mobilité, que ce soit dans le domaine professionnel (notamment pour ceux qui réalisent des tournées), ou dans le domaine privé : par exemple on n'est plus obligé de rester ou de rentrer à la maison pour recevoir un coup de fil (Ling et Yttri, 2002). On peut enfin attendre de la mobilité équipée et de son aspect rassurant pour les parents qu'elle encourage le processus d'autonomisation des jeunes, et en particulier qu'elle rende leur mobilité plus libre.

2) L'importance d'une analyse des formes différenciées d'hybridation de l'espace selon les lieux

La mise en continuité relative de l'espace pour une interaction donnée dépend des possibilités offertes par les lieux, notamment par ceux qui ne sont pas fixes (les lieux de travail, d'étude et de résidence étant les lieux fixes essentiels). Ces lieux offrent à la fois des moyens de se déplacer (ils sont situés le long des trajets) et des possibilités de connexion. Ce sont d'un côté les moyens de transport lorsque l'interaction a lieu durant le déplacement, de l'autre des lieux où séjournent temporairement les individus lors de leurs déplacements (gares, aéroports, cafés, hôtels, centres commerciaux, lieux de loisirs...) (Rallet et Torre, 2008). L'équipement différencié de l'espace détermine les formes possibles de co-présence en un lieu. Cet équipement dépend de l'évolution technologique (développement de réseaux sans fil alternatifs, convergence fixe/mobile) et de la concurrence sur les marchés de communication. Il faut aussi s'attendre à ce que des offres de mise en continuité de l'espace apparaissent (la convergence fixe/mobile en est une forme élémentaire). Cela facilitera le chaînage des activités disjointes. Sinon les individus devraient eux-mêmes «bricoler » ce chaînage, ce qui constituerait un obstacle important à son développement.

3) La décomposition spatio-temporelle des activités implique la mise au point de conventions sociales qui seront longues à se mettre en place

L'appariement entre des lieux différenciés et des activités décomposées ne peut résulter que d'un long processus d'apprentissage social. Car il implique la mise en œuvre de conventions et de pratiques sociales qui n'existent pas encore, et qui sont de même nature que les codes ayant permis d'instituer la conversation à distance par téléphone alors que cette pratique était initialement irreprésentable car totalement abstraite (au point que les usages potentiels cités de cette innovation au début du téléphone n'en faisaient pas mention). C'est ensuite que l'on peut discerner des effets sur la géographie des activités et des interactions. 
Tant que les codes sociaux nécessaires à la mise en continuité de l'espace (communiquer dans la rue ou dans un lieu public en est un) n'auront pas été élaborés, l'impact des TIC et des réseaux mobiles de communication resteront inscrits dans les pratiques antérieures et seront faiblement porteurs de changements. C'est pourquoi il convient de repérer et d'identifier les nouveaux codes sociaux d'usage des outils de communication en mobilité. L'usage différencié du téléphone portable par rapport au téléphone fixe, l'invention d'un nouveau mode de communication comme les SMS mais aussi le déploiement des mini-ordinateurs portables sur les terrasses de café ont impliqué et impliquent de nouveaux codes. Plus l'interaction est complexe (notamment lorsqu'il ne s'agit plus seulement de communiquer), plus la mise au point des conventions sociales est longue.

4) Certaines pratiques nouvelles émergent plus facilement car elles sont portées par la nature des outils utilisés et des activités et la simplicité des codes sociaux mobilisés

Prenons l'exemple de l'activité qui consiste à s'orienter dans l'espace. C'est une activité bien connue et bien antérieure à l'apparition des réseaux mobiles de communication, au point de paraître diviser l'humanité en deux, ceux qui savent s'orienter et ceux qui s'avouent incapables de le faire. Cette activité s'appuyait sur un certain nombre de pratiques différentes selon les individus : acheter une carte et préparer à l'avance son trajet, l'acheter en cours de route une fois que l'on est perdu, chercher et lire des panneaux, préférer demander son chemin à un passant, etc. Les technologies de pilotage des individus apparaissent (GPS pour les véhicules, GPS intégré au téléphone portable, consultation de cartes et calcul d'itinéraires via l'Internet mobile...). Elles simplifient la vie des individus en leur donnant des informations qu'il leur fallait préparer à l'avance ou collecter en cours de route. D'où le succès immédiat de cette technologie, d'autant qu'elle ne mobilise pas d'autres conventions que celles utilisées avant (savoir lire une carte) et au besoin les font disparaître si l'on se laisse guider par la voix. En particulier, l'usage de la technologie ne bute pas sur des codes sociaux complexes touchant aux interactions sociales puisqu'il s'agit d'un dialogue homme/machine. Les usages innovants (au sens d'avoir un impact sur notre navigation spatio-temporelle) qui s'imposeront les premiers seront ceux qui n'impliquent pas la mise au point de codes sociaux complexes, au moins dans la phase d'émergence où nous sommes. C'est pourquoi l'analyse des services de géolocalisation s'avère un objet de recherche intéressant.

5) La réalisation des potentialités offertes par les outils est subordonnée à l'acceptabilité de la mise en continuité spatio-temporelle de l'espace

Gardons nous de croire que les potentialités deviennent ipso facto des réalités.

Tout d'abord, l'existence des outils mobiles et d'un espace équipé ne signifie pas que les individus vont modifier leurs activités. Ce n'est pas parce qu'on a un téléphone ou un ordinateur portables qu' on va se mettre à aller travailler régulièrement dans un café wifi. Ce n'est pas non plus parce qu'on a un téléphone portable doté d'un accès à Internet que l'on va compulsivement s'absorber dans la navigation sur Internet ou regarder sa série TV préférée dans les transports en commun. Ce n'est pas non plus parce qu'il existe des ordinateurs portables que les employés sédentaires vont se transformer en employés nomades. De même peut-on être sceptique sur l'hypothèse d'Urry (2005) selon laquelle le temps de transport devient un temps utile, actif grâce aux outils mobiles de communication. Les outils offrent une telle possibilité mais elle est plus ou moins exploitée selon les conditions du transport et les préférences des individus. Ceux qui profitaient du temps de transport pour dormir ou se divertir continueront de le faire tandis que ceux qui n'ont de cesse d'allumer leur netbook pour travailler une fois assis étaient déjà des travailleurs acharnés lors de leurs déplacements. Il est vrai qu'il y aura des inflexions et qu'il faut les repérer mais les programmes d'activité sont rythmés par des normes sociales et institutionnelles fortes (Green, 2002). Des études 
(Lenz et Nobis, 2007) montrent par ailleurs que la recomposition des activités autour des outils ne concerne que quelques cadres hypermobiles. Ces catégories sont souvent faussement érigées en modèles censés s'imposer ensuite à tout le monde.

Mais l'obstacle principal à la mise en continuité de l'espace est son acceptabilité sociale. La connexion et la joignabilité permanentes conduisent à saturer l'espace : plus une minute, plus un lieu où l'on peut se soustraire aux autres (Aguiléra et al., 2009; Licoppe et Heurtin, 2001). Par ailleurs, la géolocalisation peut représenter une intrusion dans la vie privée. Car elle conduit à pouvoir être tracée soit par un opérateur qui exploite les données de mobilité et de localisation, soit par les membres de son réseau professionnel, amical ou familial qui peuvent connaître votre position. En réaction, certains individus se ménagent ou souhaitent se ménager des zones et des temps de déconnexion (Jauréguiberry, 2006). Mais tout le monde n'a pas les mêmes marges de manœuvre, surtout ceux qui sont placés dans des positions de subordination hiérarchique. L'algorithmisation (mise sous contrainte temporelle) qui s'est imposée aux organisations depuis les années 80 s'est étendue aux individus, y compris aux espaces et temps qu'ils étaient seuls à contrôler. Il est vraisemblable que là aussi de nouveaux codes sociaux devront être mis en place pour que la mise en continuité spatiotemporelle de l'espace ne conduise pas à une mise sous tension (et notamment sous surveillance) excessive de nos vies quotidiennes.

\section{Conclusion}

Nous avons vu que la question de savoir si les TIC avaient un effet sur la mobilité des individus était une question légitime mais un peu vaine. Primo, les TIC permettent d'économiser certains déplacements mais en induisent d'autres, de sorte que l'effet net n'est pas clair. Secundo, la mobilité répond à des déterminants puissants qui ne sont pas affectés par le développement des TIC ou en tous cas pas sous la forme d'une suppression des déplacements. Les fréquences, les raisons, les modes et les parcours des déplacements peuvent varier mais les déplacements demeurent. Enfin les relations entre TIC et déplacements ne peuvent être pensées indépendamment du contexte organisationnel, économique ou sociétal dans lequel elles fonctionnent comme moyen de coordination.

Nous avons plaidé dans la seconde partie de l'article pour un déplacement de la question des relations entre TIC et mobilité. Elle est de savoir si les TIC modifient les pratiques de mobilité, leurs formes, leur contenu plutôt que leur nombre. Pour cela, nous avons adopté une problématique où les relations entre TIC et mobilité passent par le filtre de l'analyse de pratiques et d'activités équipées par des outils mobiles de communication. Que deviennent ces activités ainsi équipées ? Pour y répondre, nous avons introduit l'idée qu'il n'y a qu'un seul espace et non deux (physique et virtuel). L'espace offre un ensemble de lieux d'où l'on peut accéder à des moyens de communications et à des connexions virtuelles. C'est un composé hybride de lieux physiques et de relations virtuelles dont il faut analyser la combinaison. Le développement des réseaux mobiles de communication se traduit par un espace fortement maillé de ces combinaisons hybrides. Ce maillage permet une certaine mise en continuité de l'espace alors que la discontinuité était une de ses caractéristiques majeures. Cela conduit à porter l'attention non sur le nombre de déplacements mais sur ce que l'on fait en situation de mobilité ou plus largement à la façon dont on agence ces activités dans le temps et dans l'espace.

Des transformations de la mobilité auront lieu mais elles sont soumises à un certain nombre de conditions. La plus importante est que soient mises en place les conventions sociales nécessaires à l'adoption de nouvelles pratiques d'interactions équipées par des outils portables de communication. C'est un processus long à se réaliser mais dont dépendront les 
transformations majeures de la mobilité. Pour l'instant, ces technologies servent surtout à optimiser la gestion de la mobilité existante. Il n'y a pas de grands bouleversements, du moins des bouleversements qui soient à la hauteur de la fièvre technologique qui accompagne la diffusion de ces outils. Mais déjà de nouvelles pratiques apparaissent comme celle de s'orienter différemment dans l'espace car les conventions qui président à cet usage ne sont pas très complexes.

\section{Références citées}

AGUILERA A., GUILLOT C., BONIN O., 2009, Mobile phone use and the management of individual reachability, Conférence of the COST 298 project, "The good, the bad and the challenging", Copenhague, mai.

ARNFALK P., KOGG B., 2003, Service transformation- managing a shift from business travel to virtual meetings. Journal of Cleaner Production,vol.11, n8, p. 859-872.

ASCHER F., 1995, Métapolis ou l'avenir des villes, Paris, Odile jacob.

BELTON L., DE CONINCK F., 2006, « Des frontières et des liens. Les topologies du privé et du professionnel pour les travailleurs mobiles”, Réseaux, n¹40, p. 67-100.

CHANDLER A.D., 1956, «Management decentralization : an historical analysis », Business History Review, vol.30, n², p.111-174.

CLAISSE G., ROWE F., 1993, «Téléphone, communication et sociabilité: des pratiques résidentielles différentiées », Sociétés Contemporaines, $n^{\circ}$ 14-15, p.. 165-189.

DOUMA F., WELLS K., HORAN T.A., KRIZEK K.J., 2004, ICT and travel in the twin cities metropolitan area: enacted patterns between Internet use and working and shopping trips. Paper presented at the 83rd Annual Meeting of Transportation Research Board, Washington, D.C., January.

DUPUY G., 1995, Les territoires de l'automobile, Economica, Paris.

FARAG S., SCHWANEN T., DIJST M, FABER J., 2007, "Shopping online and/or in store? A structural equation model of the relationships between e-shopping and in-store shopping", Transportation Research Part A, vol.41, p.125-141

GOLOB T.F, REGAN A.C., 2001, "Impacts of information technology on personal travel and commercial vehicle operations: Research challenges and opportunities", Transportation Research, C - Emerging Technologies, $\mathrm{n}^{\circ}$ 9, p.87-121.

GREEN N., 2002, "On the Move. Technology, Mobility and the Mediation of Social Time and Space", The Information Society, vol. 18, n 4, p. 281-292.

GUILLAUME M., 2000, «La nouvelle sociabilité des hypervilles. Les technologies rendent les mégapoles plus urbaines ? », La recherche, Paris, Ministère de la recherche.

HÄGERSTRAND T., 1970, «What about people in regional science? », Papers of the Regional Science Association, n²4, pp.7-21.

JAURÉGUIBERRY F., 1994, «De l'appel au local comme effet inattendu de l'ubiquité médiatique », Espaces et Sociétés, $n^{\circ}$ 74-75 «les nouveaux territoires de la prospective ».

JAURÉGUIBERRY F., 2006, «De la déconnexion aux TIC comme forme de résistance à l'urgence », Communication et organisation, $n^{\circ} 29$, Bordeaux, p. 195-203. 
KRIZEK K.J., JOHNSON A., 2003, Mapping the terrain of Information and Communication Technology (IC) and household travel, Conference of the Transportation Research Board, Washington, D.C., January.

LARSEN J. URRY J., AXHAUSEN K.W., 2007, Networks and Tourism. Mobile Social Life. Annals of Tourism Research, vol.34, ${ }^{\circ} 1$, p.244-262.

LICOPPE C., HEURTIN J.P., 2001, "Managing one's availability to telephone communication through mobile phones: a French case study of the development dynamics of mobile phone use", Personal and Ubiquitous Computing, vol. 5, n 2, p. 99-108.

LENZ B., NOBIS C., 2007, "The changing allocation of activities in space and time by the use of ICT- Fragmentation as a new concept and empirical results", Transportation Research Part A, vol. 41, n² 2, p. 190-204.

LETHIAIS V., AGUILERA A., 2007, Les relations de coopération inter-entreprises: TIC versus face-à-face. Conference Online Services : networks, contents, usages, 13-14 décembre, Paris, France.

LING R., HADDON L., 2003, Mobile Telephony, Mobility and the Coordination of Everyday Life, in Katz, J. (Ed) Machines that Become Us: The Social Context of Personal Communication Technology, Transaction Publishers, New Brunswick, New Jersey, p. 245266.

MASSOT M.H., 1995, Transports et télécommunications, Editions Paradigme.

MOELLER M., 2006, Evaluation des modèles d'affaire et des trajectoires technologiques pour l'Internet mobile, Thèse de doctorat en sciences économiques, Université de Paris Dauphine.

MOKHTARIAN P., 2000, Telecommunications and Travel. Transportation Research Board.

MOKHTARIAN P.L., SALOMON I., HANDSY S.L., 2006, “The impacts of ICT on leisure activities: A conceptual exploration”, Transportation, vol.33, n³, p. 263-289.

MUSSO P., 2008, Territoires et cyberspace en 2030, La Documentation Française, Paris.

RALLET A., 2000, «Communication à distance : au-delà des mythes », Sciences Humaines, $\mathrm{n}^{\circ} 104$, p. 26.30 .

RALLET A., 2001, "Commerce électronique et localisation urbaine des activités commerciales", Revue Economique, 52, p. 267-288.

RALLET A., 2005, "La mobilité urbaine comme facteur structurant du commerce électronique", in Moati P., Nouvelles technologies et modes de vie, Ed de l'Aube, p. 221 239.

RALLET A. ET TORRE A., 2008, Temporary Geographical Proximity for Business and Work Coordination: When, How and Where?, Working paper.

SCHWANEN T., KWAN M.P., 2007, "The Internet, Mobile phone, and space-time constraints", Geoforum, vol.39, n³, p. 1362-1377.

URRY J., 2005, Sociologie des mobilités. Une frontière pour la sociologie ?, A. Colin, Paris. 